\title{
Review of: "Safety and efficacy of laparoscopic repeat liver resection and re-operation for liver tumor"
}

Bin Jin

Potential competing interests: The author(s) declared that no potential competing interests exist.

This article incorporates numerous patient data and provides strong evidence for the evaluation of the effectiveness and safety of LRLR. 